\title{
Early Top-Down Modulation in Visual Word Form Processing: Evidence From an Intracranial SEEG Study
}

\author{
Yi Liu, ${ }^{1}$ Gaofeng Shi, ${ }^{2}$ Mingyang Li, ${ }^{1}$ Hongbing Xing, ${ }^{2}$ Yan Song, ${ }^{1}$ Luchuan Xiao, ${ }^{1}$ Yuguang Guan, ${ }^{3}$ and \\ (D)Zaizhu Han ${ }^{1}$ \\ ${ }^{1}$ State Key Laboratory of Cognitive Neuroscience and Learning and IDG/McGovern Institute for Brain Research, Beijing Normal University, Beijing \\ 100875, China, ${ }^{2}$ Faculty of International Education of Chinese Language, Beijing Language and Culture University, Beijing 100083, China, and \\ ${ }^{3}$ Department of Neurosurgery, Sanbo Brain Hospital, Capital Medical University, Beijing 100093, China
}

Visual word recognition, at a minimum, involves the processing of word form and lexical information. Opinions diverge on the spatiotemporal distribution of and interaction between the two types of information. Feedforward theory argues that they are processed sequentially, whereas interactive theory advocates that lexical information is processed fast and modulates early word form processing. To distinguish between the two theories, we applied stereoelectroencephalography (SEEG) to 33 human adults with epilepsy (25 males and eight females) during visual lexical decisions. The stimuli included real words (RWs), pseudowords (PWs) with legal radical positions, nonwords (NWs) with illegal radical positions, and stroked-changed words (SWs) in Chinese. Word form and lexical processing were measured by the word form effect (PW versus NW) and lexical effect (RW versus PW), respectively. Gamma-band $(60 \sim 140 \mathrm{~Hz})$ SEEG activity was treated as an electrophysiological measure. A word form effect was found in eight left brain regions (i.e., the inferior parietal lobe, insula, fusiform, inferior temporal, middle temporal, middle occipital, precentral and postcentral gyri) from $50 \mathrm{~ms}$ poststimulus onset, whereas a lexical effect was observed in five left brain regions (i.e., the calcarine, middle temporal, superior temporal, precentral, and postcentral gyri) from $100 \mathrm{~ms}$ poststimulus onset. The two effects overlapped in the precentral $(300 \sim 500 \mathrm{~ms})$ and postcentral $(100 \sim 200 \mathrm{~ms}$ and $250 \sim 600 \mathrm{~ms})$ gyri. Moreover, high-level regions provide early feedback to word form regions. These results demonstrate that lexical processing occurs early and modulates word form recognition, providing vital supportive evidence for interactive theory.

Key words: broadband gamma activity; feedforward theory; interactive theory; stereoelectroencephalography; visual word recognition

Significance Statement

A pivotal unresolved dispute in the field of word processing is whether word form recognition is obligatorily modulated by high-level lexical top-down information. To address this issue, we applied intracranial SEEG to 33 adults with epilepsy to precisely delineate the spatiotemporal dynamics between processing word form and lexical information during visual word recognition. We observed that lexical processing occurred from $100 \mathrm{~ms}$ poststimulus presentation and even spatiotemporally overlapped with word form processing. Moreover, the high-order regions provided feedback to the word form regions in the early stage of word recognition. These results revealed the crucial role of high-level lexical information in word form recognition, deepening our understanding of the functional coupling among brain regions in word processing networks.

Received Aug. 28, 2020; revised May 4, 2021; accepted May 9, 2021.

Author contributions: M.L., H.X., and Z.H. designed research Y.L., G.S., and Y.G. performed research; Y.L., G.S., M.L., Y.S., and L.X. analyzed data; Y.L., Y.G., and Z.H. wrote the paper.

This work was supported by the National Key Research and Development Program of China (2018YFC1315200), the National Natural Science Foundation of China (31872785, 81972144, 31871099), and the National Defense Basic Scientific Research Program of China (2018110B011). We thank the reviewers for comments and Meng Zhao and Zhao Liu of Sanbo Brain Hospital for data collection.

The authors declare no competing financial interests.

Correspondence should be addressed to Zaizhu Han at zzhhan@bnu.edu.cn or Yuguang Guan at ygguan2000@163.com.

https://doi.org/10.1523/JNEUROSC1.2288-20.2021

Copyright $\odot 2021$ the authors

\section{Introduction}

Visual word recognition entails several components, including the processing of word form (e.g., word length, letter order, and radical position) and high-level lexical information (e.g., phonology and semantics; Carreiras et al., 2014), which is supported by a wide variety of brain regions (Coltheart et al., 2010; Woollams et al., 2011). Visual word form information is coded in the left ventral occipitotemporal cortex (Binder et al., 2006; Vinckier et al., 2007; Bruno et al., 2008; Woolnough et al., 2021), whereas nonvisual lexical information is processed in multiple cortices (e.g., semantics, anterior temporal gyrus, inferior frontal gyrus, angular gyrus, posterior middle temporal gyrus; phonology, 
supramarginal gyrus, superior temporal gyrus, perisylvian cortex; Lau et al., 2008; Wu et al., 2012; Chen et al., 2020; Ding et al., 2020).

However, the spatiotemporal distribution of and interaction between the above-mentioned processing of visual word form and high-level lexical information remain unclear. Feedforward theory argues that lexical properties are processed after visual word form and do not affect word form processing (Jobard et al., 2003; Levy et al., 2009; Solomyak and Marantz, 2009). However, interactive theory assumes that visual words automatically activate top-down knowledge (e.g., sounds and meanings), which provides predictive feedback for word form processing (Woodhead et al., 2014; Whaley et al., 2016; Li et al., 2020). To solve this dispute, when and where the visual word form and nonvisual high-level lexical information are processed need to be elucidated.

Researchers have simultaneously manipulated word form and lexical factors to explore the spatiotemporal distributions of visual and nonvisual information using event-related potentials (ERPs; Hauk et al., 2006; Lin et al., 2011). For example, studies about Chinese characters used this approach. A Chinese compound character comprises a semantic radical and a phonetic radical, which are positioned following orthographic rule (Li and Kang, 1993; Shu et al., 2003). Previous studies have found that orthographically legal characters rather than orthographically illegal characters, regardless of lexicality, induced a stronger negative ERP component at $\sim 170 \mathrm{~ms}$ (N170) in the left posterior electrodes; thus, the authors inferred that visual radical position information rather than lexical features was processed at $170 \mathrm{~ms}$ after stimulus presentation (Lin et al., 2011; Yum et al., 2015, 2017). However, others have observed that phonology was already accessed at $100 \mathrm{~ms}$ in high-order regions [e.g., the left inferior frontal gyrus (IFG) and precentral gyrus (PrCG)] for English words (Wheat et al., 2010; Klein et al., 2015). The inconsistent findings about whether lexical information has been processed in the visual form processing stage might be because of the differences between languages (e.g., Chinese vs English) or between brain regions of interest (e.g., posterior primary regions vs high-order regions). To address this issue, it is necessary to inspect more precise spatiotemporal dynamics and functional connectivity between word form and lexical processes across primary and high-level regions in a given language.

The present study seeks to reveal the spatiotemporal distribution and the functional coupling of a wide range of cortical areas between word form and lexical processes during recognizing a Chinese word. To accomplish this, 33 adults with epilepsy performed a classical lexical decision task (Dehaene and Cohen, 2011), which contained real words (RWs), orthographically legal pseudowords (PWs) without meaning, orthographically illegal nonwords (NWs), and stroke-changed words (SWs; Fig. 1A). Word form processing was measured by the word form effect (i.e., $\mathrm{PW}$ vs NW), whereas lexical processing was measured by the lexical effect (i.e., RW vs PW), with the power of broadband gamma activity (BGA; $60 \sim 140 \mathrm{~Hz}$ ) of participants' intracranial stereoelectroencephalography (SEEG) signals as an electrophysiological measure. Different from the ERPs originating from a low-frequency oscillation $(<40 \mathrm{~Hz})$, the BGA is highly correlated with the firing rates of local neuronal populations, reflecting increases (or decreases) in neural activity (Lachaux et al., 2012), particularly in advanced cognitive tasks (e.g., language and memory; Kadipasaoglu et al., 2014; Cibelli et al., 2015).

\section{Materials and Methods}

\section{Participants}

Thirty-three adults with epilepsy (25 males) were recruited from Sanbo Brain Hospital, Capital Medical University, China. They were stereotactically implanted with depth electrodes to localize seizure foci for further clinical treatment. An a priori power analysis was conducted with $\mathrm{G}^{\star}$ Power 3.1.7 ( $F$ tests, ANOVA repeated measures, within factors; Faul et al., 2007) based on the criterion ( $\alpha=0.05$; effect size $f=0.25)$. The effect size was estimated according to two relevant previous studies (Lin et al., 2011; Wang et al., 2016). The power analysis indicated that 24 participants in total would ensure $80 \%$ statistical power. All were native Chinese Mandarin speakers and most (29 patients) were right-handed (Oldfield, 1971). The patients' mean age and education duration were 27.48 years $(\mathrm{SD}=6.26$; range, $18 \sim 43$ years $)$ and 12.15 years $(\mathrm{SD}=2.87$; range, $6 \sim 17$ years), respectively. The seizure zones in these patients covered the bilateral frontal, parietal, temporal, and occipital lobes and central sulcus (Table 1). The patients were implanted with 354 electrodes with 5035 contacts in total. All but six patients had their electroencephalography (EEG) signals recorded from 64 contacts using the 64-channel EEG system by BrainAmp amplifiers (Brain Products; Table 1). A contact was not selected to record when it was the ending contact of the electrode, showed high impedance $(>15 \mathrm{k} \Omega)$, or was close to the epileptogenic zone. The epileptogenic zone of each patient was identified from preoperative magnetic resonance imaging (MRI), positron emission tomography (PET), and SEEG recordings. We also attempted to choose contacts in classical language areas that were repeatedly reported to participate in language processing, such as Broca's area, Wernicke's area, the fusiform gyrus (FG), the inferior temporal gyrus (ITG), and the precentral gyrus (PrCG; Jobard et al., 2003; Bolger et al., 2005; Ferstl et al., 2008; Price, 2012; Wu et al., 2012). The six patients who did not have all 64 contacts recorded had fewer than 64 contacts meeting the above inclusion criteria. Thus, 2095 contacts from 332 electrodes were recorded in our experiment. More electrodes (left 232 vs right 100) and contacts (1456 vs 639) were located in the left hemisphere (Fig. 2A; Table 1). The patients provided informed written consent. The study was approved by the Institutional Review Board of the National Key Laboratory of Cognitive Neuroscience and Learning, Beijing Normal University.

\section{Materials and experimental procedure}

The stimuli had 300 Chinese single-character words, including 150 RWs and 150 false words. False words did not exist in the Chinese corpus, including 50 PWs, 50 NWs, and 50 SWs. An RW (e.g., “种”, plant,/ zhong4/) has a semantic (S) radical (“禾”, crops,/he2/) and a phonetic (P) radical (“中”, middle,/zhong1/). The semantic radical usually provides semantic clues for the whole word, whereas the phonetic radical provides phonological clues (Weekes et al., 2006; Bi et al., 2007). Radicals usually have certain positions in Chinese characters (Taft et al., 1999). PW's or NW's S and P radicals appeared in their commonly or unusually occupied positions, respectively. SW was created by adding or subtracting one stroke from an RW (Fig. 1A). RWs and false words had the same radicals. In other words, false words were created by combining the radicals of the RWs. A classical lexical decision task was adopted. Each word was presented for $1000 \mathrm{~ms}$, interspersed with an unfixed-duration interstimulus interval (ISI, $1500 \sim 2000 \mathrm{~ms}$ ). During the ISI, a fixation cross, which was used to direct the participants' attention and to obtain a low-level baseline for neural activity, was presented for 1000 $1500 \mathrm{~ms}$, followed by a $500 \mathrm{~ms}$ blank screen (Fig. 1B). The participants were instructed to decide whether the word was real by pressing the Yes or No button using their right hand. Half of the patients pressed the Yes button with their index finger and the No button with their middle finger (labeled as the yes-index finger group), whereas the other half pressed the buttons in the reverse (the yes-middle finger group). The experiment comprised 10 blocks, each having 30 stimuli. Seventeen patients (patient codes $1 \sim 3,6,8,9,13 \sim 17,24,26 \sim 28,31$, and 32 in Table 1) completed the task twice. When participants conducted the task, the intracranial SEEG signals were recorded on-line at a sampling rate of $5000 \mathrm{~Hz}$ by BrainAmp amplifiers. 


\section{A Stimuli example}

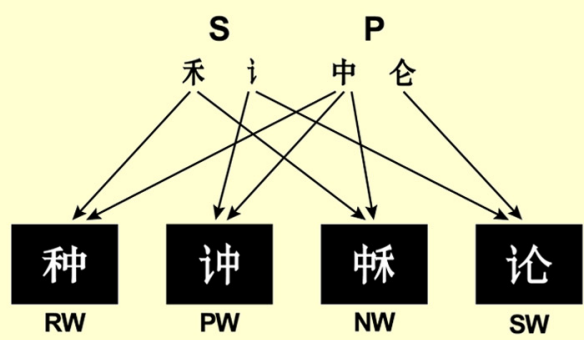

\section{B Experimental procedure}

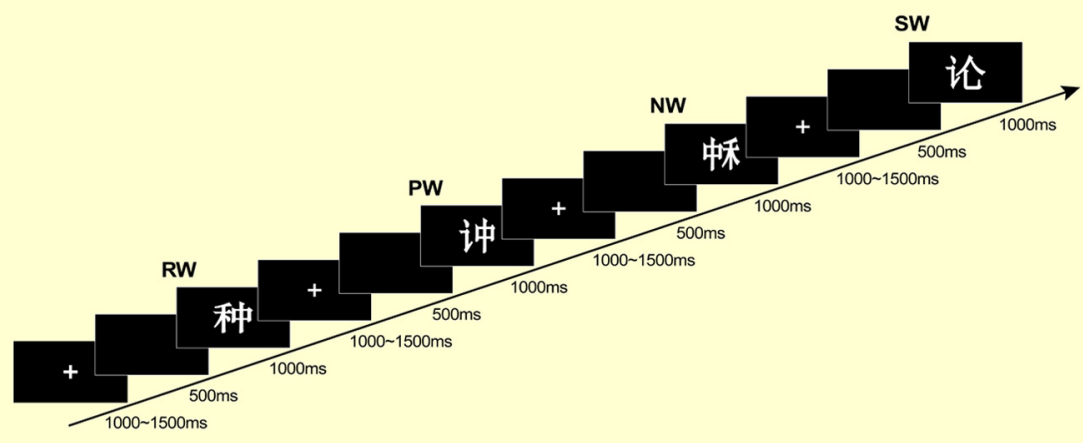

\section{Behavioral result}
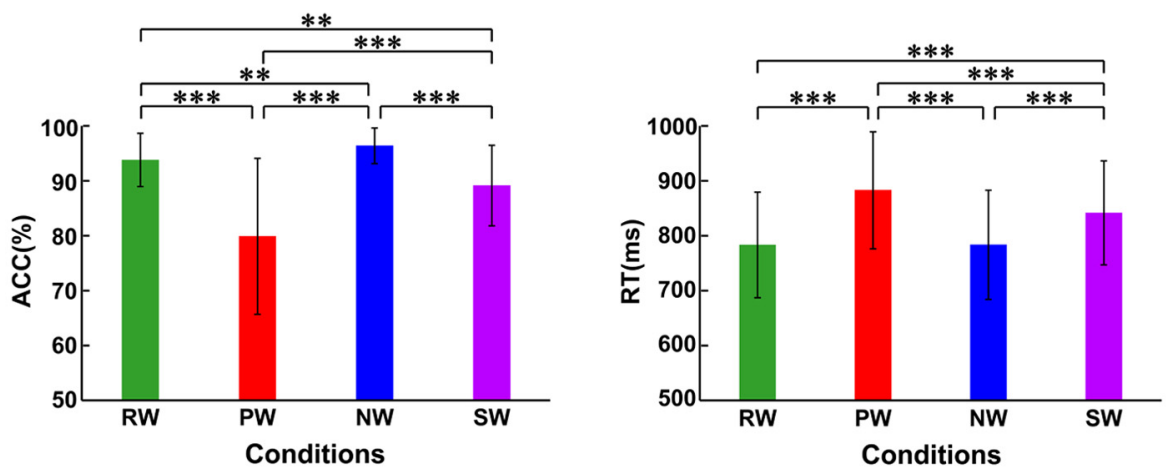

Figure 1. Experimental stimuli, procedure, and behavioral results. $\boldsymbol{A}$, Stimuli example. Each stimulus consists of a semantic radical and a phonetic radical. $\boldsymbol{B}$, Experimental procedure. The stimuli were presented randomly. C, Behavioral result. Error bars represent SDs; ${ }^{* *} p<0.01$, Bonferroni corrected; ${ }^{* * *} p<0.001$, Bonferroni corrected.

\section{Behavioral analysis}

We calculated patients' accuracy (ACC) and reaction time (RT) in the four experimental conditions (RW, PW, NW, and SW). One-way repeated ANOVA was used to analyze the main effects of the condition, and a paired-sample $t$ test was used to compare the difference between conditions (Bonferroni corrected $p<0.01$ ).

\section{SEEG data preprocessing}

SEEG signals were processed using the EEGLAB toolbox (Delorme and Makeig, 2004), the FieldTrip toolbox (Oostenveld et al., 2011), and inhouse scripts in MATLAB 2018b (MathWorks). Data processing consisted of the following steps. (1) The raw EEG signals were referenced on-line to a scalp contact placed at the vertex and rereferenced to the average of the remaining contacts off-line after removing the contacts that displayed high impedance $(>15 \mathrm{k} \Omega)$ and contained many artifacts or epileptiform activities. (2) The data were filtered off-line with a finite impulse response filter from 1 to $200 \mathrm{~Hz}$, notch filtered at $50 \mathrm{~Hz}$ and its harmonics to remove line noise, downsampled to $1000 \mathrm{~Hz}$, and segmented in -1 to $2 \mathrm{~s}$ epochs relative to the stimulus onset. (3) Epochs followed by incorrect behavioral responses were excluded. To control for the potential artifacts in the raw SEEG data, we further removed the epochs where most contacts showed improbable values (4 SDs away from the mean amplitudes across all the epochs), and those whose voltages were $>350 \mu \mathrm{V}$. 4). To obtain the stimulus-related oscillations, we conducted time-frequency analysis to calculate the event-related spectral perturbation (ERSP), which indicated the correlation of the changes in the power spectrum with the onset time of the experimental stimulus (Makeig et al., 2004). In response to the stimulus presentation, an increase or a decrease in EEG oscillations in specific frequency bands was defined as event-related synchronization (ERS) or desynchronization (ERD), respectively. The time-frequency decomposition for each epoch was performed in the frequency band of interest (high $\gamma$ frequency band, $60 \sim 140 \mathrm{~Hz}$ ) through a Morlet-based wavelet transform. The width of each window in each wavelet transform was seven cycles. 
Table 1. Background information of 33 patients with epilepsy

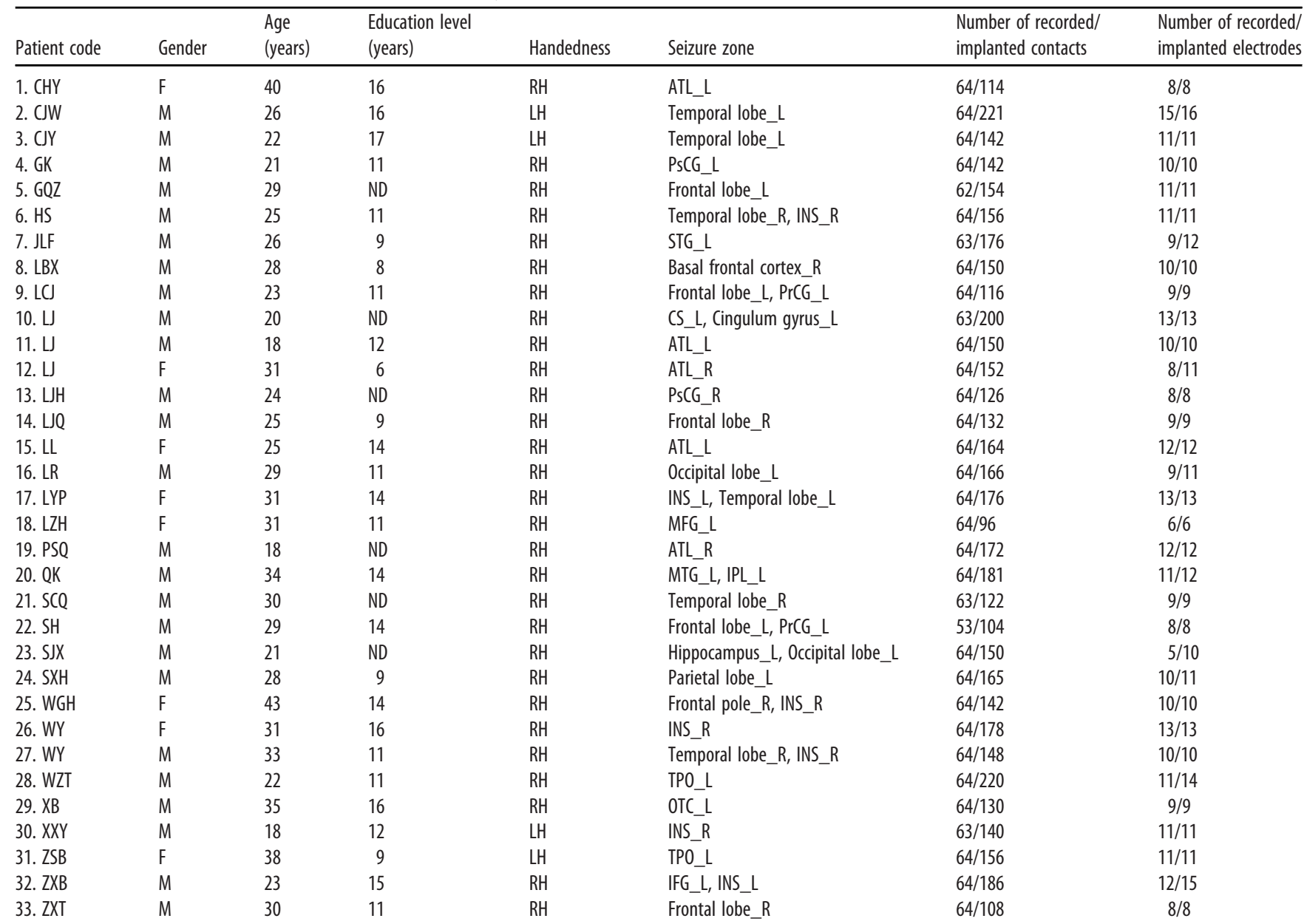

Handedness was assessed by Edinburgh Handedness Inventory (Oldfield, 1971), and seizure zone was localized by the gathered data, including the preoperative MRI, PET, and SEEG recordings. M, Male; F, female; LH, lefthanded; RH, right-handed; L, left hemisphere; R, right hemisphere; ATL, anterior temporal lobe; OTC, occipitotemporal cortex; TP0, temporo-parieto-occipital junction.

To correct for the rapid dropoff in the spectral power with frequency, the power of each epoch was normalized to the inverse square frequency (Thesen et al., 2012). The power at each time frequency point after the stimulus presentation was normalized to the average power at baseline (from $800 \mathrm{~ms}$ before stimulus presentation to the stimulus onset) using $z$-scores. The converted absolute values were averaged over the frequency band and the epochs. (5) To localize the anatomic positions of intracranial contacts for each patient, the brain images in the preoperative MRI scans were registered to those in the postoperative computer tomography scans using FreeSurfer (Fischl, 2012). To perform group analysis across all patients (Babajani-Feremi et al., 2016), we projected the contacts from each subject onto a standard Montreal Neurologic Institute (MNI) reference brain to gain the MNI coordinates of all contacts. Using BrainNet Viewer software (Xia et al., 2013), the contacts from all patients were superimposed on a brain surface template for visualization. Further analyses were restricted to the contacts located in the Anatomical Automatic Labeling (AAL) gray matter template (Tzourio-Mazoyer et al., 2002).

\section{Behavior-SEEG power mapping analysis}

Identifying the task-relevant regions for collapsed experimental conditions. To define the brain regions that were involved in visual word recognition, we first selected the task-responsive contacts that were activated in the lexical decision task. In all trials of RW, PW, NW, and SW conditions, a contact was defined as responsive if it induced significant ERS or ERD in BGA, which exceeded the mean \pm 3 SDs of BGA values during the baseline period (an $800 \mathrm{~ms}$ time window before stimulus onset) for a consecutive $50 \mathrm{~ms}$. The $50 \mathrm{~ms}$ time window provides an estimate of significant BGA activation that is less susceptible to momentary fluctuations (Osman et al., 1992; Matsuo et al., 2015; Ozker et al., 2017). Contact selection was performed for each time point from stimulus onset to $800 \mathrm{~ms}$ poststimulus presentation. It is worth noting that the identified contacts might engage in whole visual lexical recognition processing, including word-specific processes and cognition-general processes (e.g., visual perception, working memory, decision-making, and executive control). Then, we divided these responsible contacts into anatomic brain regions based on the AAL cerebral atlas. A brain region was defined as task related if it contained at least 20 responsible contacts from at least five different patients.

To further examine whether response fingers affected the SEEG result pattern, we calculated the incidence of task-relevant patients in the yes-index finger group (or the yes-middle finger group) in each task-relevant region. Namely, the number of task-relevant yes-index finger (or yes-middle finger) patients divided by the total number of patients in the yes-index finger (or the yes-middle finger). The incidences between the two finger groups were compared with $\chi^{2}$ tests.

Finally, we further extracted the curves of BGA power over time for all contacts in each task-relevant region. The following two measurements were calculated to describe the dynamic characteristics of these curves. The onset latency was defined as the earliest time point when the BGA power after stimulus presentation rose above (or fell below) baseline noise for $50 \mathrm{~ms}$ and was estimated for each responsive contact using the trial-averaged BGA power. The peak latency was defined as the duration time from the stimulus onset to the time where the contact obtained the maximum BGA value (peak power) before the end of the entire epoch (2000 ms after stimulus presentation). Independent sample $t$ tests 


\section{A Anatomical location of all 2095 contacts from 33 patients}


$\mathbf{R}$

प⿴⿱卄一二八土口丩

$1 \quad$ Patient code 33

\section{B Anatomical location of 399 selected task-relevant contacts from 23 patients}
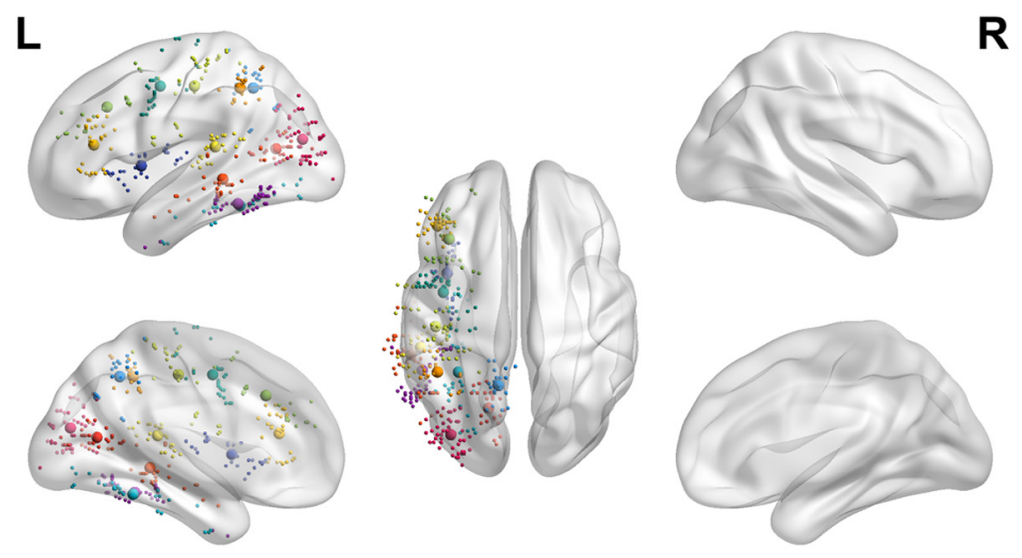

$\mathbf{R}$
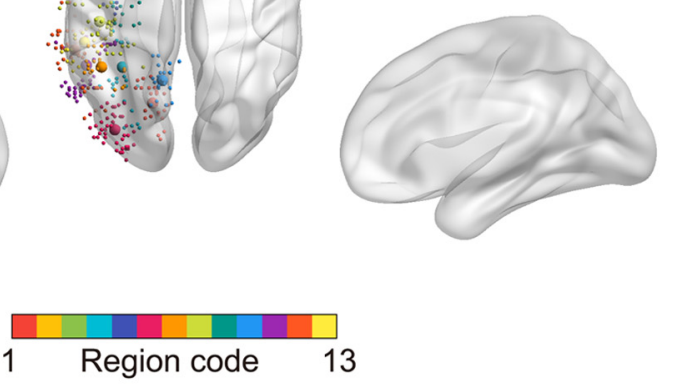

Figure 2. Contact distribution of patients. A, Anatomical location of all 2095 contacts from 33 patients. The contacts of a patient are coded in the same color. $\boldsymbol{B}$, Anatomical location of 399 selected task-relevant contacts from 23 patients. The contacts in a region are coded in the same color. The large dot in a region presents the averaged location of the contacts in this region.

were conducted across all contacts for each pair of brain regions to investigate whether differences existed in the onset latencies (or peak latencies) of the contacts among these regions [false discovery rate (FDR) corrected $p<0.01]$.

Delineating the activity of each experimental condition in task-relevant regions. To examine which task-relevant regions were causally involved in each experimental condition (RW, PW, NW, and SW), we conducted the following analyses. First, we averaged the power values of contacts in each brain region for each patient in successive time windows (time window length, $100 \mathrm{~ms}$; sliding time, $50 \mathrm{~ms}$ ). Based on previous EEG studies on word recognition processing (Hauk et al., 2006; Chen et al., 2013; Hirshorn et al., 2016; Woolnough et al., 2021), we only analyzed the EEG data before $600 \mathrm{~ms}$ poststimulus presentation. Second, we calculated the inverse efficiency (IE) of each patient in each brain region. The IE measure referred to the average response time of correct trials divided by accuracy, which was used to reflect the inversed behavioral efficiency (Townsend and Ashby, 1983; Wei et al., 2012). Third, we computed Spearman's rank correlations between the power value and IE across the patients $(p<0.05)$. Finally, confirmatory Bayesian statistical analyses for all correlations were performed using Kendall's tau-b and the default settings in JASP version 8.6 software (https://jasp-stats.org/). Bayes factors $\left(\mathrm{BF}_{10}\right)$ were used to index how likely the two variables should be correlated (Wagenmakers et al., 2018).

Revealing the difference between experimental conditions in each task-relevant region. The present study focuses on delineating the spatiotemporal dynamics of the word form effect (PW vs NW) and lexical effect (RW vs PW) in each task-related region. However, the results of the contrast between RW and NW conditions (i.e., word form plus lexical effect) could also provide an additional comprehensive interpretation for the function of each region. Therefore, we compared the difference 
in SEEG power values among the three conditions (RW, PW, and NW) in each region. Specifically, we ran a linear mixed-effects model for each task-relevant brain region in successive time windows (time window length, $100 \mathrm{~ms}$; sliding time, $50 \mathrm{~ms}$ ) before $600 \mathrm{~ms}$ poststimulus presentation (FDR corrected $p<0.05$ ). In the model, we tested for fixed effects of experimental condition with the BGA power values as the dependent variable and behavioral IE as a covariate. When a region showed a significant main effect in the model, a pairwise comparison was conducted between the conditions in the region (FDR corrected $p<0.05$ ). Thus, we extracted the exact time windows of the three effects: the word form effect (PW vs NW), lexical effect (RW vs PW), and word form plus lexical effect (RW vs NW). A conjunction analysis further distinguished the same and different time windows among the effects in each region.

To further validate the significant effects observed in this analysis, we calculated the proportion of contacts whose effect tendency was consistent with that of the observed significant word form (PW vs NW), lexical (RW vs PW), or word form plus lexical effect (RW vs NW).

Investigating the feedback received by the left ventral occipitotemporal and occipital cortices from other task-relevant regions in each experimental condition. To explore the direction of information flow between different brain regions, we performed Granger causal analysis (GCA) with a multivariate autoregressive model. GCA assumes that all time courses have covariance stationarity, and if the values of past time point (s) of time course $X$ can forecast the current values of time course $Y$, then $X$ causally modulates $Y$. This approach has been widely used with intracranial EEG data (Perrone-Bertolotti et al., 2012; Si et al., 2017). To improve the stationarity of the data, we conducted the following steps: detrending, first-order differencing, and subtracting the mean voltages from the preprocessed data. The Dickey-Fuller test $(\alpha=0.01)$ was applied to test the unit roots. The optimal model order was determined by evaluating the Akaike information criterion (Akaike, 1974) and the Bayesian information criterion (Seth, 2005). However, similar to previous electrophysiological studies, both criteria failed to yield an optimal model order (e.g., Brovelli et al., 2004; Gow et al., 2008; Gow and Segawa, 2009; Omigie et al., 2015). Therefore, we further tested three other model orders $(15,20,25)$ and obtained similar results for these orders. To simplify, we only reported the results of a model order of 25 . The Granger causal (GC) values were computed with a $200 \mathrm{~ms}$ window size during $400 \mathrm{~ms}$ prestimulus onset to $1000 \mathrm{~ms}$ poststimulus onset. They were further normalized to the $400 \mathrm{~ms}$ prestimulus baseline by percentage change (\%). The statistical significance of GC values was obtained via surrogate statistics (permutation test with 200 permutations). If the GC values between two brain regions in a patient were significantly higher than those of the random distribution over $50 \mathrm{~ms}(p<$ $0.05)$, connectivity between the regions was considered to exist for this patient. To avoid false positive results, we regarded the existence of a connection between two brain regions in the patient group only if its connectivity strength was significant in more than half the patients. Note that the connectivity analysis could only be conducted in patients who had contacts in two regions of interest at the same time. Among the regions showing word form effects, the left ventral occipitotemporal cortex (VOTC) plays a key role in processing orthographic information (e. g., bigram frequency, number of common letters, consonant-vowel structure; Vinckier et al., 2007; Lochy et al., 2018; Woolnough et al., 2021). In addition, the left occipital cortex has also been involved in orthographic tasks (Wu et al., 2012) and associated with visual word form representations (Zhang et al., 2018). Therefore, we examined the directional connectivity to VOTC [i.e., fusiform gyrus (FG) and inferior temporal gyrus (ITG)] and occipital cortex (i.e., MOG) from other taskrelevant brain regions. They included the left calcarine sulcus [CS: 7 patients $\rightarrow$ FG (the number of patients who could be used for conducting GCA from CS to FG); $5 \rightarrow$ ITG; $6 \rightarrow$ MOG], IFG $(3 \rightarrow$ FG; $2 \rightarrow$ ITG; $4 \rightarrow$ MOG), inferior parietal lobe (IPL: $3 \rightarrow$ FG; $3 \rightarrow$ ITG; $4 \rightarrow$ MOG), insula (INS: $3 \rightarrow$ FG; $2 \rightarrow$ ITG; $3 \rightarrow$ MOG), middle frontal gyrus (MFG: $2 \rightarrow$ FG; $2 \rightarrow$ ITG; $2 \rightarrow$ MOG), middle temporal gyrus (MTG: 8 $\rightarrow$ FG; $7 \rightarrow$ ITG; $9 \rightarrow$ MOG), precuneus (PcUN: $4 \rightarrow$ FG; $4 \rightarrow$ ITG; $5 \rightarrow$ MOG), postcentral gyrus (PsCG: $3 \rightarrow$ FG; $2 \rightarrow$ ITG; $5 \rightarrow$ MOG), PrCG $(1 \rightarrow$ FG; $1 \rightarrow$ MOG), and superior temporal gyrus (STG: $7 \rightarrow$ FG; $6 \rightarrow$ ITG; $7 \rightarrow$ MOG).

\section{Data availability}

The datasets that support the findings of this study are available from the corresponding authors on request.

\section{Results}

\section{Behavioral performance}

The behavioral performance of 33 subjects in the lexical decision task is shown in Fig. $1 C$. The main effect of ACC among the four conditions (RW, PW, NW, and SW) was significant $\left(F_{(3,96)}=\right.$ 32.40; $p<0.001 ; \eta_{\mathrm{p}}{ }^{2}=0.50$; one-way ANOVA). The ACC of the PW condition $[0.80$ (mean) $\pm 0.14(\mathrm{SD})]$ was significantly lower than that of the other three conditions: RW $(0.94 \pm 0.05$; $t_{(32)}=-5.26$; Bonferroni corrected $p<0.001$; Cohen's $d=$ -0.92 ; paired $t$ test $), \mathrm{NW}\left(0.96 \pm 0.03 ; t_{(32)}=-7.25\right.$; corrected $p<0.001$; Cohen's $d=-1.26$; paired $t$ test), and SW (0.89 \pm $0.07 ; t_{(32)}=-5.38$; corrected $p<0.001$; Cohen's $d=-0.94$; paired $t$ test). The ACC of the SW condition was significantly lower than that of the NW condition $\left(t_{(32)}=-5.86\right.$; corrected $p<0.001$; Cohen's $d=-1.02$; paired $t$ test) and the RW condition $\left(t_{(32)}=-3.23\right.$; corrected $p=0.003$; Cohen's $d=-0.56$; paired $t$ test). Furthermore, the ACC of the NW condition was significantly higher than that of the RW condition $\left(t_{(32)}=2.97\right.$; corrected $p=0.006$; Cohen's $d=0.52$; paired $t$ test). Similarly, the main effect of RT among the four conditions was significant $\left(F_{(3,96)}=59.40 ; p<0.001 ; \eta_{\mathrm{p}}^{2}=0.65\right.$; one-way ANOVA). Follow-up pairwise comparisons showed that the RT of the PW condition ( $882.70 \mathrm{~ms} \pm 106.4 \mathrm{~ms}$ ) was significantly longer than that of the RW condition $\left(783.20 \mathrm{~ms} \pm 96.21 \mathrm{~ms} ; t_{(32)}=\right.$ 8.20; corrected $p<0.001$; Cohen's $d=1.43$; paired $t$ test), the $\mathrm{NW}$ condition $\left(783.50 \mathrm{~ms} \pm 99.30 \mathrm{~ms} ; t_{(32)}=15.21\right.$; corrected $p<0.001$; Cohen's $d=2.65$; paired $t$ test), and that of the SW condition $\left(841.50 \mathrm{~ms} \pm 94.53 \mathrm{~ms} ; t_{(32)}=4.80\right.$; corrected $p<$ 0.001 ; Cohen's $d=0.84$; paired $t$ test). The RT of the SW condition was significantly longer than that of the NW condition $\left(t_{(32)}=9.73\right.$; corrected $p=0.001$; Cohen's $d=1.69$; paired $t$ test) and the RW condition $\left(t_{(32)}=6.26\right.$; corrected $p<0.001$; Cohen's $d=1.09$; paired $t$ test). However, there was no significant difference in RT between the RW and NW conditions $\left(t_{(32)}=-0.03\right.$; corrected $p=0.98$; Cohen's $d=-0.005$; paired $t$ test). Generally, the patients performed more poorly in the PW condition than in the other three conditions.

\section{SEEG analysis results}

Task-relevant regions in collapsed experimental conditions

We recorded EEG signals from 2095 contacts in 33 patients (Fig. $2 A$; Table 1). To perform SEEG analyses, we removed the following six types of recorded contacts successively: (1) those with much high electric resistance during the experiments (in the left hemisphere, 28; right, 11), (2) those with many artifacts or epileptiform activities (left, 75; right, 20), (3) those with $>10 \%$ improbable trials of all the trials (left, 104; right, 35), (4) those beyond the gray matter AAL regions (Tzourio-Mazoyer et al., 2002; left, 259; right, 125), (5) those unrelated to our visual lexical decision task (left, 423; right, 236), and (6) those not reaching our inclusion criteria (at least 20 task-relevant contacts from at least five different patients in an AAL brain region; left, 168; right, 212). The remaining contacts (left, 399; right, 0) were distributed in 13 regions in the left hemisphere and came from 23 patients (Fig. 2B; Table 2). These identified brain regions were considered to be task relevant and might participate in multiple processes, such as visual perception, working memory, decisionmaking, executive control, or lexical/word form processes. For each task-relevant region, the incidence of task-relevant patients 
Table 2. Mean MNI coordinates of contacts in each task-relevant region

\begin{tabular}{lll}
\hline Region (abbreviation) & Mean value $(x, y, z)$ & SD $(x, y, z)$ \\
\hline Left calcarine sulcus (CS) & $-16,-65,13$ & $4,9,4$ \\
Left fusiform gyrus (FG) & $-32,-46,-17$ & $5,22,9$ \\
Left inferior frontal gyrus (IFG) & $-43,31,15$ & $5,5,10$ \\
Left insula (INS) & $-38,6,4$ & $3,12,6$ \\
Left inferior parietal lobe (IPL) & $-43,-46,45$ & $8,7,6$ \\
Left inferior temporal gyrus (ITG) & $-51,-45,-16$ & $8,17,7$ \\
Left middle frontal gyrus (MFG) & $-37,24,35$ & $8,16,11$ \\
Left middle occipital gyrus (MOG) & $-36,-79,18$ & $6,8,11$ \\
Left middle temporal gyrus (MTG) & $-57,-37,-3$ & $8,17,11$ \\
Left precuneus gyrus (PCUN) & $-11,-53,45$ & $5,8,12$ \\
Left precentral gyrus (PrCG) & $-40,-4,46$ & $9,9,12$ \\
Left postcentral gyrus (PsCG) & $-43,-22,46$ & $10,12,15$ \\
Left superior temporal lobe (STG) & $-51,-33,14$ & $8,8,6$ \\
\hline
\end{tabular}

was insignificant in the yes-index finger and yes-middle finger groups ( $p s>0.05 ; \chi^{2}$ tests). The results indicated that the pressing button fingers did not influence the probability of task-relevant occurrence.

The contacts in each of the 13 task-relevant regions showed significant ERS of BGA values when the trials of four experimental conditions (RW, PW, NW, and SW) were averaged (Fig. 3A). Note that most of these task-relevant regions (10 regions) could also be defined even when only using trials at the first time of the task. Additionally, the Pearson correlations between the time series of the trials in the first time and those in the two times were also high in these 10 regions (all correlation coefficients $>0.97$; $p s<0.001)$. Thus, we only reported the results at both times throughout this article.

To elucidate the spatiotemporal interrelationship between task-relevant regions, we compared the response onset latencies and peak latencies of the BGA time series among the regions. The onset latencies of three posterior visual regions (the left FG, $142 \mathrm{~ms} \pm 55 \mathrm{~ms}$; ITG, $265 \mathrm{~ms} \pm 186 \mathrm{~ms}$; MOG, $197 \mathrm{~ms} \pm$ $171 \mathrm{~ms}$ ) were earlier than those of 3 regions (the left CS, $421 \mathrm{~ms}$ $\pm 164 \mathrm{~ms}$; INS, $422 \mathrm{~ms} \pm 174 \mathrm{~ms}$; STG, $550 \mathrm{~ms} \pm 207 \mathrm{~ms} ; t$ values -10.04 to -3.45 ; FDR corrected $p s<0.01$; Cohen's $d$ values -2.84 to -0.86 ; independent $t$ tests). The onset latencies of two posterior visual regions (the left FG and MOG) were earlier than those of four regions (the left IFG, $333 \mathrm{~ms} \pm 151 \mathrm{~ms}$; IPL, $334 \mathrm{~ms}$ $\pm 152 \mathrm{~ms}$; PcUN, $366 \mathrm{~ms} \pm 140 \mathrm{~ms}$; and PsCG, $357 \mathrm{~ms} \pm$ $179 \mathrm{~ms} ; t$ values -7.76 to -3.08 ; FDR corrected $p s<0.01$, Cohen's $d$ values -2.26 to -0.78 ; independent $t$ tests). However, the onset latencies of two posterior visual regions (the left ITG and MOG) were comparable with those of the remaining three regions (the left MFG, $227 \mathrm{~ms} \pm 103 \mathrm{~ms}$; MTG, $256 \mathrm{~ms} \pm$ $174 \mathrm{~ms}$; and PrCG, $260 \mathrm{~ms} \pm 158 \mathrm{~ms}$; $t$ values -0.96 to 1.68 ; FDR corrected $p s>0.05$; Cohen's $d$ values -0.24 to 0.39 ; independent $t$ tests; Fig. $3 B$ ). The peak latencies of the three posterior visual regions (the left FG, $355 \mathrm{~ms} \pm 325 \mathrm{~ms}$; ITG, $433 \mathrm{~ms} \pm$ $379 \mathrm{~ms}$; and MOG, $373 \mathrm{~ms} \pm 381 \mathrm{~ms}$ ) were not significantly different ( $t$ values -0.89 to 0.73 , FDR corrected $p s>0.05$; Cohen's $d$ values -0.22 to 0.16 ). The peak latencies of the three regions were significantly earlier than those of the other six regions (the left CS, $945 \mathrm{~ms} \pm 503 \mathrm{~ms}$; INS, $795 \mathrm{~ms} \pm 392 \mathrm{~ms}$; IPL, $742 \mathrm{~ms} \pm$ 328 ms; PcUN, $801 \mathrm{~ms} \pm 471 \mathrm{~ms}$; PsCG, $705 \mathrm{~ms} \pm 274 \mathrm{~ms}$; and $\mathrm{STG}, 761 \mathrm{~ms} \pm 269 \mathrm{~ms} ; t$ values -5.12 to -3.74 ; FDR corrected $p$ values $<0.01$; Cohen's $d$ values -1.44 to -0.81 ; independent $t$ tests) but were comparable with those of three brain areas (the left MFG, $525 \mathrm{~ms} \pm 386 \mathrm{~ms}$; MTG, $411 \mathrm{~ms} \pm 351 \mathrm{~ms}$; and PrCG, $536 \mathrm{~ms} \pm 295 \mathrm{~ms} ; t$ values -2.29 to -0.26 ; FDR corrected $p s>$ 0.05 ; Cohen's $d$ values -0.59 to -0.06 ; independent $t$ tests; Fig.
$3 C)$. Only one of the occipitotemporal regions reached peak power earlier than the remaining region (the left MOG vs the left IFG, $679 \mathrm{~ms} \pm 487 \mathrm{~ms} ; t_{(73)}=3.05$; FDR corrected $p<0.01$; Cohen's $d=0.71$; independent $t$ test). These results indicate that some high-order brain regions (e.g., the left MFG, MTG, and PrCG) might be coactivated in parallel with the posterior ventral occipitotemporal and occipital cortices during visual word processing.

Activity of each experimental condition in task-relevant regions Figure 4 shows the correlations between the power of BGA of each task-related region and the behavioral IE of each experimental condition across patients. Significant correlations appeared in two regions for the RW condition (the left INS, $100 \sim 200 \mathrm{~ms}$ and $150 \sim 250 \mathrm{~ms}$; left PcUN, $200 \sim 300 \mathrm{~ms}$ and $350 \sim 600 \mathrm{~ms}$; $p s<0.05$ ), two regions for the PW condition (the left IPL, $0 \sim 100 \mathrm{~ms}$ and left MTG, $50 \sim 150 \mathrm{~ms}$; $p s<0.05$ ), two regions for the NW condition (the left INS, $400 \sim 550 \mathrm{~ms}$ and left PcUN, $450 \sim 600 \mathrm{~ms} ;$ ps $<0.05$ ), and one region for the SW condition (the left CS, $150 \sim 250 \mathrm{~ms}$; $p<0.05)$. These results demonstrate that high-level brain regions (e.g., the left INS, IPL, MTG, and PcUN) might be involved in early visual word recognition.

The difference between experimental conditions in each task-relevant region

Figure 5 shows BGA power values over time for three conditions (RW, PW, and NW) in each region. The linear mixed-effects model showed significant main effects of the experimental condition in 10 task-relevant regions ( $F$ values 4.60 to 17.27 ; FDR corrected $p s<0.05 ; \eta_{\mathrm{p}}{ }^{2}$ values 0.12 to 0.35 ; linear mixed effects). A significant word form effect (PW vs NW; FDR corrected $p s<0.05$ ) was observed in eight left task-relevant regions by pairwise comparisons (the left FG, $150 \sim 550 \mathrm{~ms}, t_{(54)}=-3.11, p=0.003$, Cohen's $d=$ -0.59 ; INS, $450 \sim 550 \mathrm{~ms}, t_{(63)}=2.14, p=0.036$, Cohen's $d=$ 0.39; IPL, $50 \sim 200 \mathrm{~ms}, t_{(56)}=-3.93, p<0.001$, Cohen's $d=-0.89$; ITG, $200 \sim 600 \mathrm{~ms}, t_{(83)}=-2.90, p=0.005$, Cohen's $d=-0.45$; MOG, $150 \sim 550 \mathrm{~ms}, t_{(90)}=-2.40, p=0.019$, Cohen's $d=-0.36$; MTG, $50 \sim 200 \mathrm{~ms}, t_{(92)}=-4.25, p<$ 0.001, Cohen's $d=-0.68$; PrCG, $300 \sim 500 \mathrm{~ms}, t_{(65)}=-3.44$, $p=0.001$, Cohen's $d=-0.60$; and PsCG, $100 \sim 200 \mathrm{~ms}$, $t_{(69)}=-3.38, p=0.001$, Cohen's $d=-0.56$ and $250 \sim$ $600 \mathrm{~ms}, t_{(71)}=-4.63, p<0.001$, Cohen's $d=-0.77$; linear mixed effects). Except for the left INS, other brain regions showed higher BGA power in the NW condition than in the PW condition. A significant lexical effect (RW vs PW; FDR corrected $p<0.05)$ was observed in five left task-related regions (the left CS, $400 \sim 600 \mathrm{~ms}, t_{(57)}=3.83, p<0.001$, Cohen's $d=0.77$; MTG, $200 \sim 300 \mathrm{~ms}, t_{(83)}=-3.31$, $p=0.001$, Cohen's $d=-0.53$; PrCG, $300 \sim 500 \mathrm{~ms}, t_{(65)}=$ $3.40, p=0.001$, Cohen's $d=0.59$; PsCG, $100 \sim 200 \mathrm{~ms}, t_{(70)}=$ 2.71, $p=0.009$, Cohen's $d=0.45$ and $250 \sim 600 \mathrm{~ms}$, $t_{(71)}=4.56, p<0.001$, Cohen's $d=0.76$; and STG, $500 \sim$ $600 \mathrm{~ms}, t_{(54)}=2.75, p=0.008$, Cohen's $d=0.56$; linear mixed effects). Except for the left MTG, other brain regions induced higher BGA power in the RW condition than in the PW condition. The word form effect had time windows identical to the lexical effect in the left PrCG $(300 \sim 500 \mathrm{~ms})$ and PsCG $(100 \sim 200 \mathrm{~ms}$ and $250 \sim 600 \mathrm{~ms})$. As the difference between the RW and NW conditions at least included both word form and lexical processes, it is not surprising that a significant difference between them also simultaneously appeared in 


\section{A Time course}

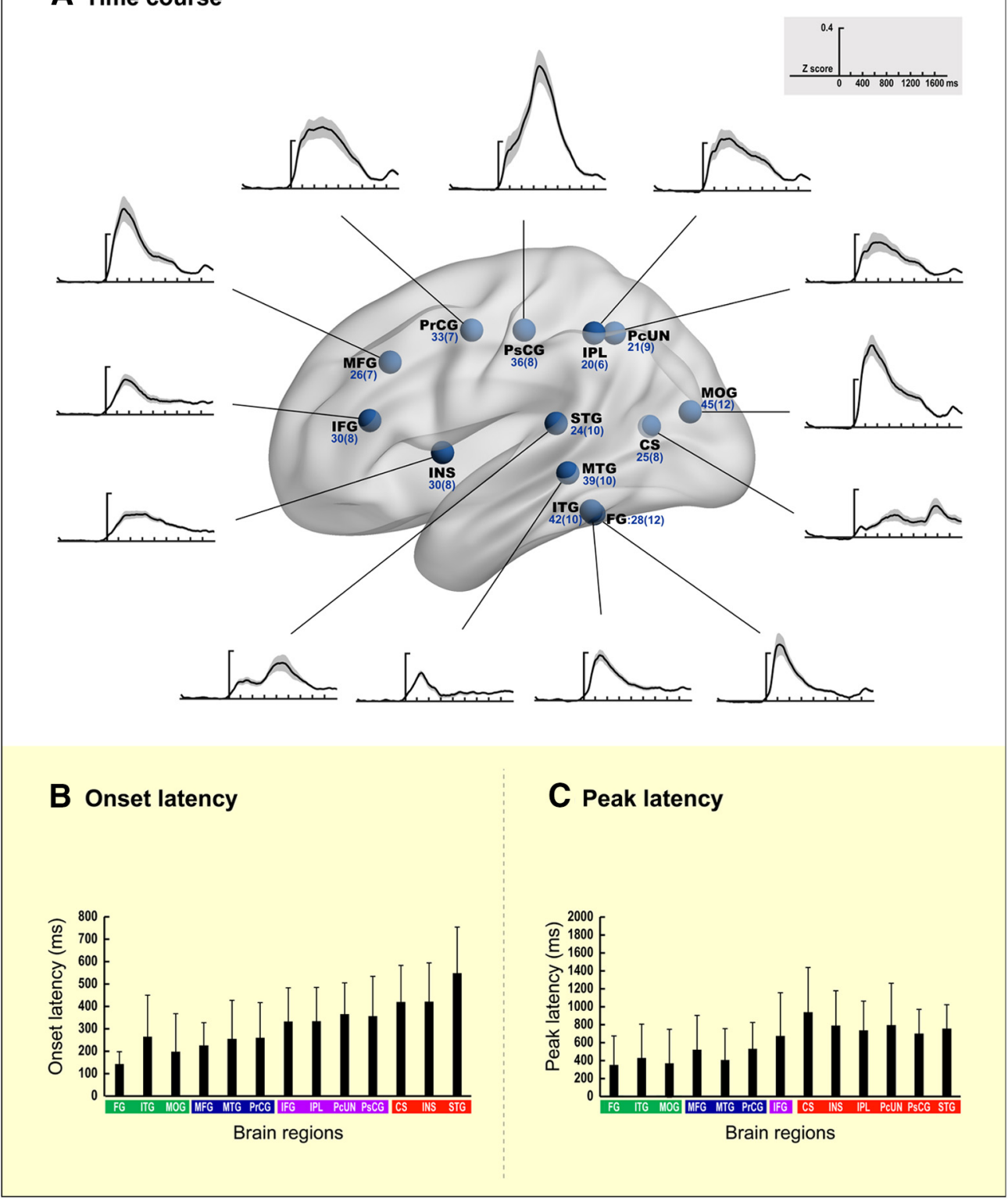

Figure 3. Time course and temporal characteristics of BGA in 13 task-relevant regions. $A$, Time course. The BGA time courses were averaged across all epochs and all contacts in each region. Shaded areas around these curves show the SEs across contacts. For display purposes, these BGA time series were temporally smoothed with a $100 \mathrm{~ms}$ boxcar filter implemented in the Fieldtrip toolbox (http://www.ru.n//donders/fieldtrip). The $x$-axis represents the time (ms) locked to stimulus onset, and the $y$-axis indicates the BGA power, which was normalized to $z$-scores relative to the baseline. The numbers of contacts and patients in a region is written in light blue, and the name of the region is written in black. $B, 0$ nset latency. The $x$-axis represents different regions, and the $y$-axis indicates the onset latency. Error bars denote SDs. All the ventral occipitotemporal and occipital regions in green rectangles showed significant BGA responses earlier than the regions in red rectangles. Two regions in green rectangles (the left FG and MOG) were activated earlier than the regions in purple rectangles. The onset latencies of activations of another two regions in green rectangles (the left ITG and MOG) were similar to those of the regions in blue rectangles. C, Peak latency. The graphic expressions are the same as those in $\boldsymbol{B}$ except that the $y$-axis shows peak latency rather than onset latency. All the ventral occipitotemporal and occipital regions in green rectangles obtained peak power earlier than the regions in red rectangles, although similar to the regions in blue rectangles. One region in the green rectangle (the left MOG) obtained peak power earlier than regions in purple rectangles. Brain regions in the same color rectangle were arranged according to the order of the initial letters of their names. The full names of the regions are shown in Table 2.

the time windows of each of the above word form effects or lexical effects. However, two of 10 regions (left PrCG, 300 $\sim 500 \mathrm{~ms}$; PsCG, $100 \sim 200 \mathrm{~ms}$ and $250 \sim 600 \mathrm{~ms}$ ) did not show a significant difference between RW and NW, although they presented both a significant word form effect and a significant lexical effect. It might reflect more working memory load when encoding unfamiliar visual representations (e.g., the NW stimuli; Rämä et al., 2001; Yang et al., 2011).

The validation analysis for the word form effect revealed that a consistent tendency appeared in a majority of contacts in each region (the left FG, 20/28 contacts; INS, 20/30; IPL, 15/20; ITG, 34/42; MOG, 33/45; MTG, 29/39; PrCG, 25/33; and PsCG, 30/36). Similarly, the lexical effects also showed consistent effect tendencies in most contacts in each region (the left CS, 18/25; MTG, 26/ 39; PrCG, 27/33; PsCG, 31/36; STG, 15/24). Consistent tendencies of the word form plus lexical effects were also found in a majority of contacts in each region (the left CS, 18/25; FG, 23/28; INS, 26/30; IPL, 18/20; ITG, 29/42; MOG, 31/45; MTG, 26/39; STG, 19/24).

These results indicate that lexical processing might occur at the early stage of word recognition (e.g., in the left MTG and 


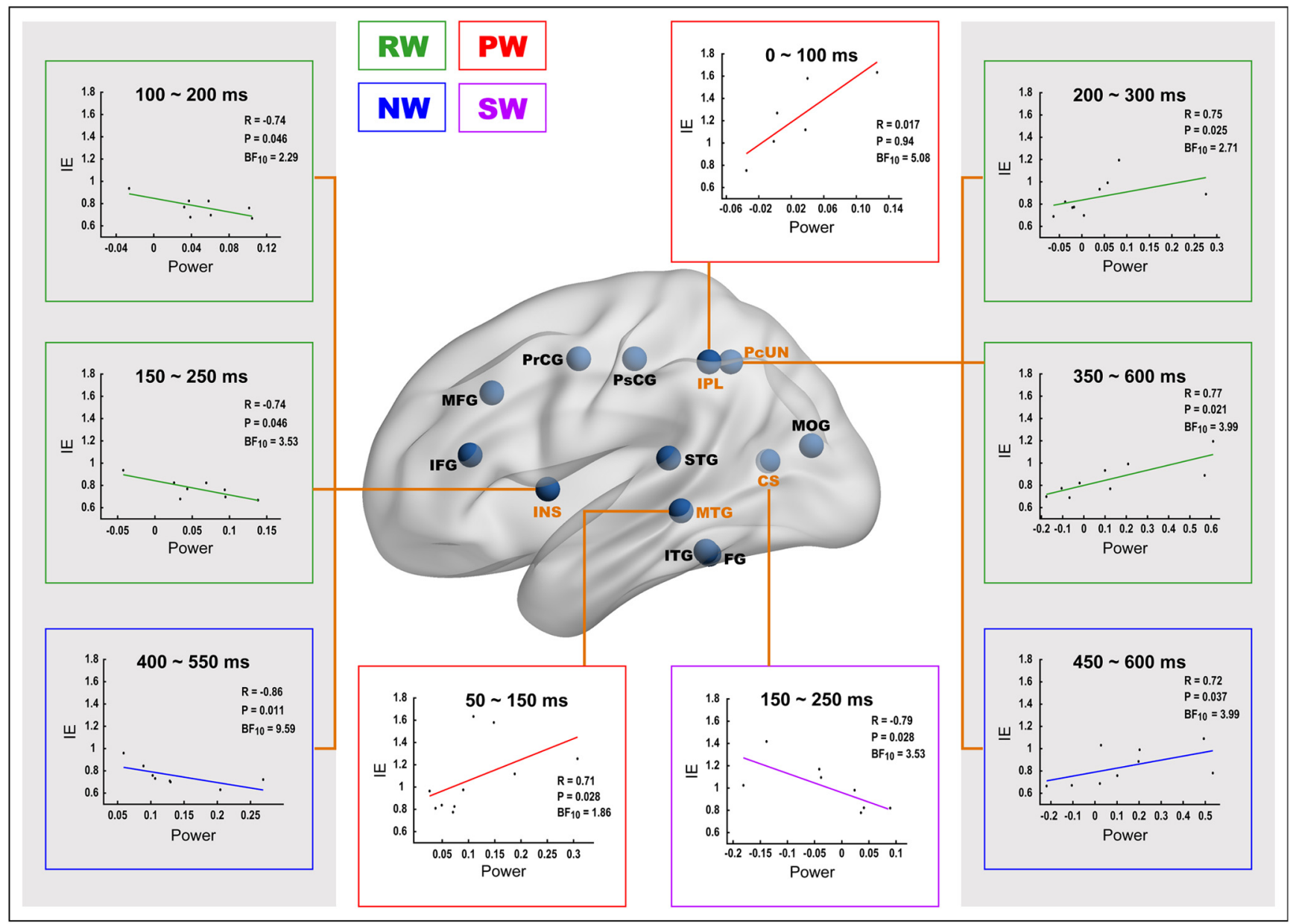

Figure 4. Significant correlation between the power of BGA of regions and the IE, the average response time of correct trials divided by accuracy, in each experimental condition across patients. This figure only shows the suprathreshold correlation diagrams. Each correlation diagram was color coded to represent its correlation condition as described by the color rectangles positioned in the top left corner. Specifically, green represents the RW condition, red represents the PW condition, blue represents the NW condition, and purple represents the SW condition. The $x$-axis represents the averaged power of a given time window, which was normalized to $z$-scores relative to the baseline, and the $y$-axis represents IE. In each diagram, R represents the Spearman correlation coefficient, $\mathrm{P}$ represents the significance level, and $\mathrm{BF}_{10}$ reflects how likely the two variables should be correlated. Correlation diagrams of one brain region were arranged in chronological order. The full names of the regions can be found in Table 2.

PsCG) from $100 \mathrm{~ms}$ after stimulus onset and that lexical processing overlays word form processing in time and space (e.g., in the left PrCG and PsCG).

The feedback received by the left ventral occipitotemporal and occipital cortices from other task-relevant regions in each experimental condition

Figure 6 illustrates the strength of the interregional connectivity from high-level task-relevant regions to the ventral occipitotemporal cortex (i.e., the left FG and ITG) and the posterior occipital cortex (i.e., the left MOG) before $150 \mathrm{~ms}$ after stimulus presentation, where a word form effect was observed in the posterior word form regions. The left MOG received directional functional connectivity from the left IFG for the RW condition; it received feedback from the left IFG, INS, IPL, and PsCG for the PW condition; and it was connected from the left INS, PcUN, and PsCG for the NW condition. The left ITG received directional connectivity from the left CS for the RW condition, and it received feedback from the left CS, IPL, MTG, and PsCG for the PW condition. The left FG received directional connectivity from the left IFG for the RW condition, it received directional connectivity from the left MTG and PsCG for the PW condition, and it received feedback from the left INS for the NW condition. These results demonstrate that word form regions (including the left FG, ITG, and MOG) received early top-down modulation from nonvisual high-level regions (e.g., the left IFG, INS, IPL, PcUN, PsCG, and MTG) in word form processing of word recognition.

\section{Discussion}

To examine whether high-level lexical information is involved in early visual word form processing, the current study compared the power of BGA $(60 \sim 140 \mathrm{~Hz})$ for four types of stimuli (RW, PW, NW, and SW) in a visual lexical decision task for 33 adults with epilepsy. Only $19.05 \%$ of the contacts (399/2095) responded to these experimental stimuli and were distributed in 13 left-brain regions. Possible reasons for the lack of task-relevant brain regions in the right hemisphere are that (1) the current task mainly depends on left-dominant processing and (2) some crucial brain tissues for the lexical decision were not recorded because of sparse sampling of SEEG or a few contacts. Among these regions, word form processing (measured by the word form effect, i.e., PW vs NW) occurred from $50 \mathrm{~ms}$ after the stimulus onset. High-level lexical processing (measured by the lexical effect, i.e., RW vs PW) occurred from $100 \mathrm{~ms}$ after the stimulus onset. The lexical effect occurred at early latency and even spatiotemporally overlapped 


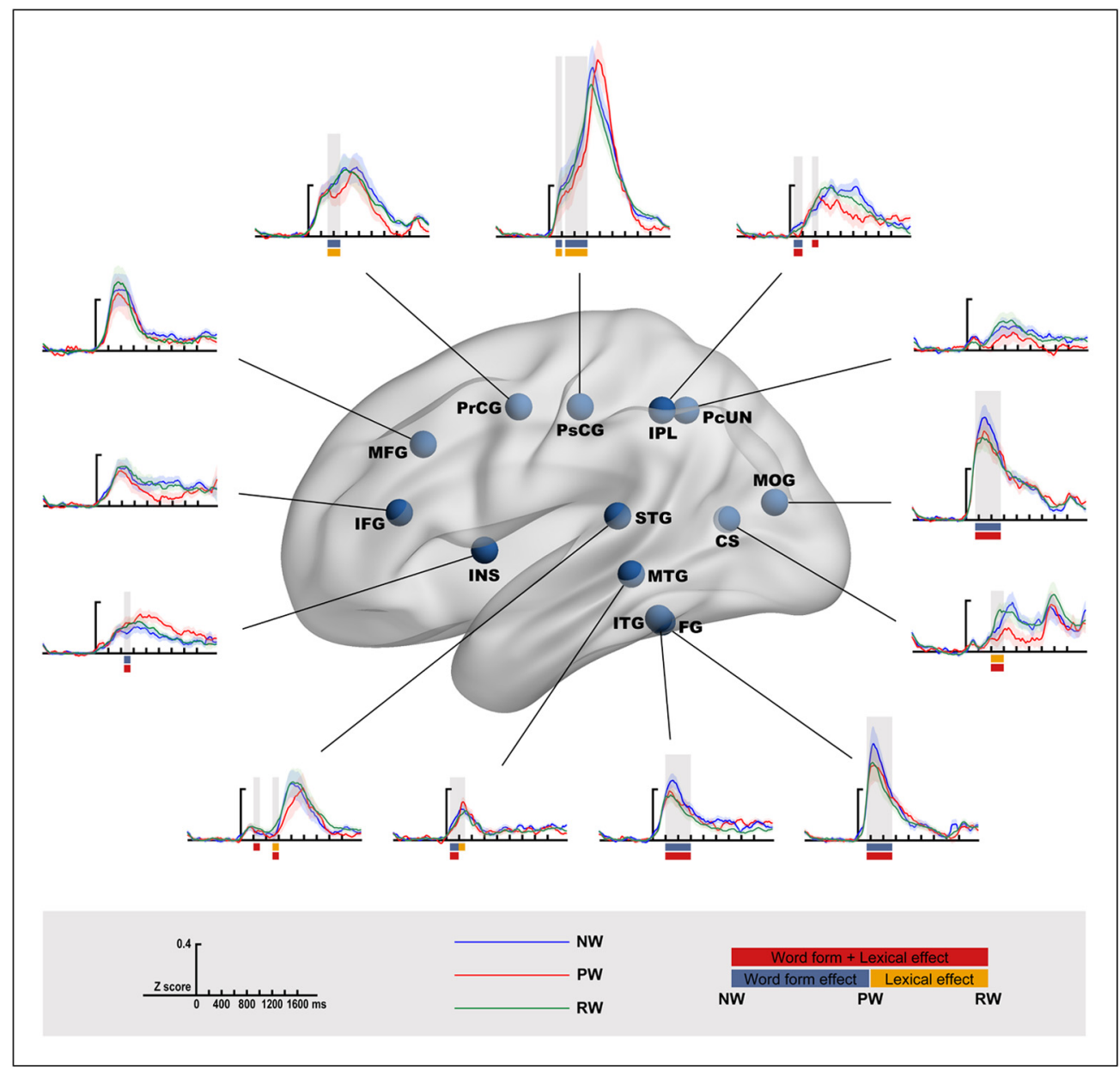

Figure 5. BGA power values of experimental conditions in each task-relevant region. Each diagram shows BGA time courses, which were normalized to $z$-scores relative to the baseline of RW, PW, and NW conditions for comparison. SEs across contacts are shaded in light colors. The $x$-axis represents time (ms) locked to stimulus onset, and $y$-axis represents the estimated BGA power values derived from the linear mixed-effects models run for each time point. The color rectangular bars below the time curves indicate significant time windows of word form effect (PW vs NW), lexical effect (RW vs PW), or word form plus lexical effect (RW vs NW) estimated by linear mixed-effect models after factoring out the IE, average response time of correct trials divided by accuracy. FDR corrected $p<0.05$. The full names of brain regions are listed in Table 2 .

with the word form effect in the left PrCG $(300 \sim 500 \mathrm{~ms})$ and PsCG $(100 \sim 200 \mathrm{~ms}$ and $250 \sim 600 \mathrm{~ms})$. Moreover, the highlevel regions provided early top-down feedback to the word from regions. This provides supporting evidence for interactive theory by investigating fine spatiotemporal dynamics of word processing across a wide range of brain areas in a given language.

\section{Word form processing regions}

We observed word form effects in eight brain regions (left FG, INS, IPL, ITG, MOG, MTG, PrCG, and PsCG) from 50 ms poststimulus presentation. We also found that among these brain regions, the activation patterns induced by the stimuli with legal word form (e.g., RW and PW) were similar, but different from the activation pattern induced by the stimuli with illegal word form (e.g., NW). The left FG and ITG, located in the VOTC, play critical roles in processing visual word form (Nobre et al., 1994; Woolnough et al., 2021) and represent orthography from coarse to fine over time (Hirshorn et al., 2016). We found a word form effect in the left VOTC from $150 \mathrm{~ms}$ poststimulus presentation, which might support gist-level discrimination of words with different visual statistics (Hirshorn et al., 2016). Previous literature also suggested that the occipital cortex showed a preference for visual words (Zhang et al., 2018) and participated in orthographic tasks of Chinese characters (Wu et al., 2012). This brain region might be responsible for visuospatial processing and ordering symbols in unfamiliar strings (Boros et al., 2016). Thus, the early activity of the MOG might contribute to the visuospatial processing of the stimuli structured by complex square-combined configurations.

The left IPL is known to play an essential role in the spatial selection, sequencing, and discrimination of spatial positions of letters (Pammer et al., 2006; Cohen et al., 2008; Ossmy et al., 2014; Carreiras et al., 2015b). Interestingly, we observed a word form effect in the left IPL starting from $50 \mathrm{~ms}$ after stimulus onset, even earlier than the word form effects observed in the VOTC and occipital cortices. We inferred that the letter position information might be transferred from the dorsal parietal areas to the ventral visual stream by potential functional and anatomic connectivity (Bouhali et al., 2014; Finn et al., 2014). The left INS was involved in word reading and contributed to phonology (Dickens et al., 2019). In the current study, we observed higher activation in the left INS for the orthographically well-formed stimuli (e.g., the RW and PW) compared with the stimuli with illegal orthography (e.g., the NW), which indicates that this region might process the abstract orthography. The left MTG, PrCG, and PsCG were found to be involved in both word form and lexical processing, and we discuss their roles in the following section. 

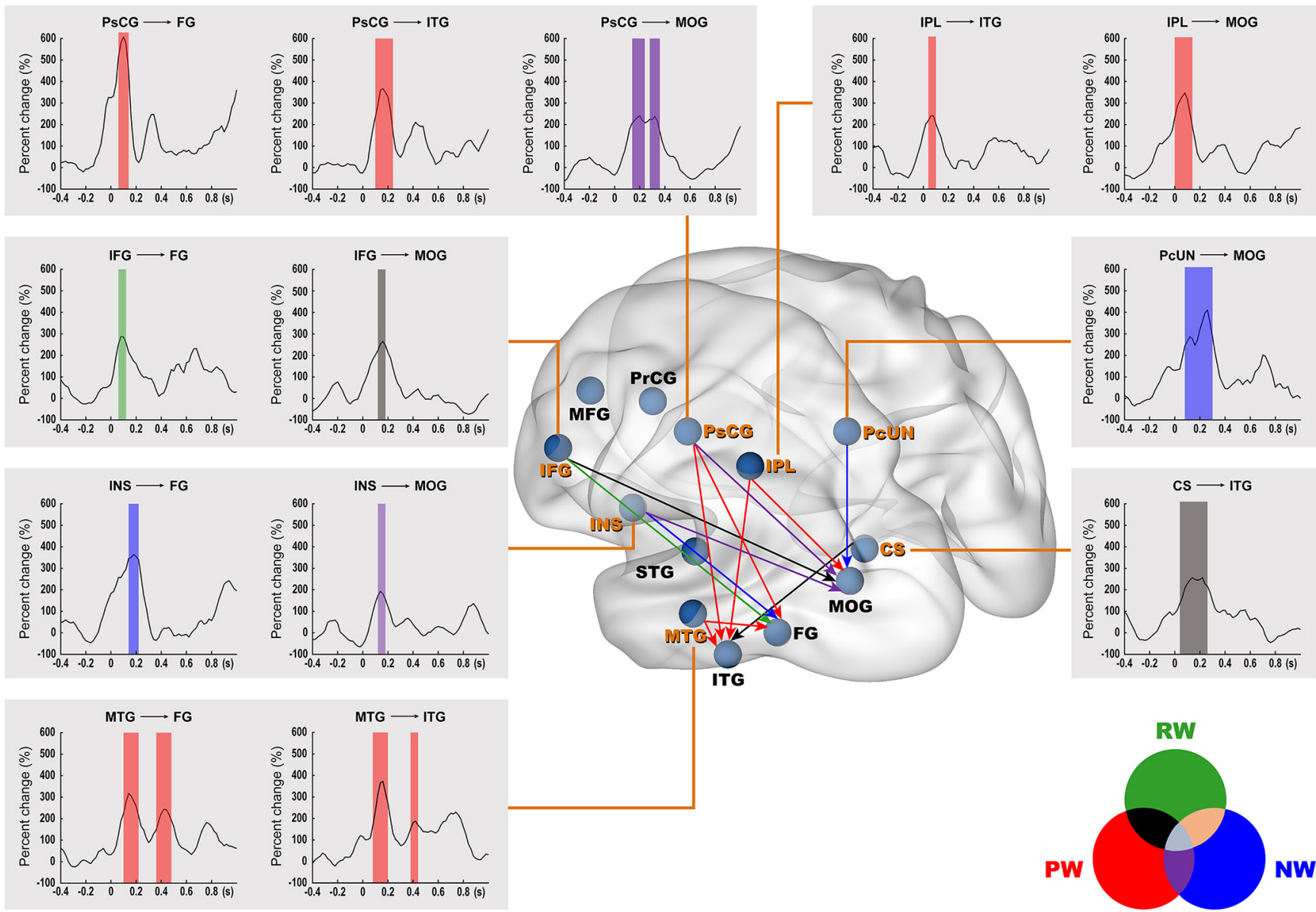

Figure 6. Directional connectivity from the high-level linguistic regions to the left ventral occipitotemporal and occipital cortices. This figure shows early directional connectivity toward the fusiform gyrus (or inferior temporal gyrus, middle occipital gyrus) that occurred before word form processing (150 ms poststimulus presentation). The color circles (bottom right) describe the relationship of the lines with arrows in different colors on the brain map. Specifically, the black lines with arrows represent the shared connectivity of the RW and PW. The purple lines with arrows represent the shared directional connectivity of the PW and NW conditions. The red lines with arrows represent the directional connectivity that only occurred in the PW condition. The blue lines with arrows represent the directional connectivity that only occurred in the NW condition. The green lines with arrows represent the directional connectivity that only occurred in the RW condition. This figure also displays several representative figures that show the early directional connectivity before $150 \mathrm{~ms}$ poststimulus presentation. The $x$-axis represents the time (s) relative to the onset time of the stimulus, and the $y$-axis represents the Granger causal connectivity values that were corrected by baseline data (the average Granger causality from -400 to $0 \mathrm{~ms}$ ) using percent change (\%). The shaded areas with translucent colors mark the time windows of the suprathreshold Granger causality, and these shaded areas were color coded in the same way that the lines with arrows were coded. The full names of the brain regions are given in Table 2.

\section{High-level linguistic regions}

We found that lexical effects occurred in five brain regions (the left CS, MTG, PrCG, PsCG, and STG) from 100 ms poststimulus presentation. Early lexical effects might not merely be driven by the adopted high-level task. Carreiras et al. (2015a) observed early activations of the left angular gyrus and intraparietal sulcus at $\sim 120 \mathrm{~ms}$ after stimulus onset even in a low-level visual task (pushing a button when a dot was presented as part of a stimulus; Carreiras et al., 2015a). The left CS is usually considered to be related to early visual processing (De Putte et al., 2018). Interestingly, we observed that this region was engaged in lexical processing. The findings might be explained by interactive theory (Price and Devlin, 2011), which emphasized that the function of the occipitotemporal region was determined by the interaction of visual input and top-down predictions induced by nonvisual attributes. The left STG is often implicated in phonological access, phonological short-term memory (Mano et al., 2013), and sublexical print-to-sound mapping (Tan et al., 2001). Compatible with the previous study (Hauk et al., 2012), the left MTG showed an early lexical effect from $200 \mathrm{~ms}$. Earlier than the lexical effect, we also observed a word form effect. The left MTG might be important in the interface of orthographic information with semantic information for reading (Purcell et al., 2014), the link between word form and semantic networks (Price and Mechelli, 2005), lexico-semantic processing (Taylor et al., 2013), and the access and retrieval of semantic information (Gitelman et al., 2005; Wei et al., 2012). The left PrCG and PsCG have been found to be associated with phonology (Dickens et al., 2019). Particularly, the left PrCG is usually dedicated to the prelexical phonological representations from orthography (Wheat et al., 2010) and articulatory mapping (Dickens et al., 2019). Notably, in the left PrCG and PsCG, no significant differences were observed for the BGA power in the RW and NW conditions. One possibility might be that encoding the less familiar NW stimuli might increase working memory load and require stronger activations of the two regions to maintain working memory during the visual lexical decision task (Rypma et al., 1999; Rämä et al., 2001).

\section{Modulation of linguistic regions to word form processing regions}

Consistent with previous electrophysiological studies (Woodhead et al., 2014; Whaley et al., 2016), the present study showed 
directional connectivity from high-order language brain regions to word form regions at early latency (before $150 \mathrm{~ms}$ poststimulus presentation). Whaley et al. (2016) discovered early modulations of the visual form region from frontal regions (e.g., the left IFG and MFG) associated with phonological representations during word production. Consistently, the current study also found that high-level brain regions (e.g., the left IFG, INS, PsCG), which were linked to graphophonological conversion and spelling-sound translation (Jobard et al., 2003; Borowsky et al., 2006), provided early feedback toward the word form brain regions. Different from the above-mentioned two studies, more semantically related brain regions (e.g., the left MTG; Binder et al., 2009; Wu et al., 2012) were connected to the ITG in the current study. Different feedback connections might indicate that top-down modulations were affected by task demands, as proposed by the interactive theory (Price and Devlin, 2011). Oral production mainly requires the articulation of phonology, whereas lexical decision needs more semantic processes (Edwards et al., 2005).

As suggested by interactive theory (Carreiras et al., 2014), we found that the top-down modulations were affected by the properties of visual input. For PW stimuli that contain phonetic radicals, more feedback from the left dorsal IPL, which might be associated with the transformation from orthography to phonology (Taylor et al., 2013) and the short-term storage of phonological information (Tan et al., 2005), modulated word form processing. For NW stimuli with novel word form, PcUN, which might be related to visuospatial imagery (Cavanna and Trimble, 2006), provided more feedback to word form processing.

\section{Limitations}

The current study has at least the following caveats. (1) Inequivalent numbers of stimuli were contained in different experimental conditions (RW, 300 trials; PW, 100 trials; NW, 100 trials; SW, 100 trials), which might bias the lexical effect (RW vs PW). (2) GCA revealed that some high-level brain regions provide feedback to the left VOTC and MOG immediately after stimulus presentation. This might be because of the short interstimulus interval (1500 $2000 \mathrm{~ms}$ ). Postreaction monitoring or predictions might produce top-down modulations that are too early. (3) Because of a lack of control task, word-specific processing could not be isolated from other general processes (e.g., visual perception, working memory, decision-making, and executive control).

\section{Conclusion}

By analyzing the SEEG signals recorded from 33 adults with epilepsy, we observed that lexical processing occurred early and even overlapped with word form processing in time and space. Furthermore, high-order brain regions (e.g., the left IFG, INS, IPL, MTG, PcUN, and PsCG) provided early top-down feedback to the primary visual word form regions (i.e., the left FG, ITG, MOG). These results demonstrate that word form and lexical processes in visual word recognition are interactive rather than sequential, providing important insights into the neural network dynamics of visual word recognition.

\section{References}

Akaike H (1974) A new look at the statistical model identification. IEEE Trans Automat Contr 19:716-723.

Babajani-Feremi A, Narayana S, Rezaie R, Choudhri AF, Fulton SP, Boop FA, Wheless JW, Papanicolaou AC (2016) Language mapping using high gamma electrocorticography, fMRI, and TMS versus electrocortical stimulation. Clinical Neurophysiology 127:1822-1836.
Bi Y, Han Z, Weekes BS, Shu H (2007) The interaction between semantic and the nonsemantic systems in reading: evidence from Chinese. Neuropsychologia 45:2660-2673.

Binder JR, Medler DA, Westbury CF, Liebenthal E, Buchanan L (2006) Tuning of the human left fusiform gyrus to sublexical orthographic structure. Neuroimage 33:739-748.

Binder JR, Desai RH, Graves WW, Conant LL (2009) Where is the semantic system? A critical review and meta-analysis of 120 functional neuroimaging studies. Cereb Cortex 19:2767-2796.

Bolger DJ, Perfetti CA, Schneider W (2005) Cross-cultural effect on the brain revisited: universal structures plus writing system variation. Hum Brain Mapp 25:92-104.

Boros M, Anton J-L, Pech-Georgel C, Grainger J, Szwed M, Ziegler JC (2016) Orthographic processing deficits in developmental dyslexia: beyond the ventral visual stream. Neuroimage 128:316-327.

Borowsky R, Cummine J, Owen WJ, Friesen CK, Shih F, Sarty GE (2006) FMRI of ventral and dorsal processing streams in basic reading processes: insular sensitivity to phonology. Brain Topogr 18:233-239.

Bouhali F, Thiebaut de Schotten M, Pinel P, Poupon C, Mangan J-F, Dehaene S, Cohen L (2014) Anatomical connections of the visual word form area. J Neurosci 34:15402-15414.

Brovelli A, Ding M, Ledberg A, Chen Y, Nakamura R, Bressler SL (2004) Beta oscillations in a large-scale sensorimotor cortical network: directional influences revealed by granger causality. Proc Natl Acad Sci U S A 101:9849-9854.

Bruno JL, Zumberge A, Manis FR, Lu ZL, Goldman JG (2008) Sensitivity to orthographic familiarity in the occipito-temporal region. Neuroimage 39:1988-2001.

Carreiras M, Armstrong BC, Perea M, Frost R (2014) The what, when, where and how of visual word recognition. Trends Cogn Sci 18:90-98.

Carreiras M, Monahan PJ, Lizarazu M, Duñabeitia JA, Molinaro N (2015a) Numbers are not like words: different pathways for literacy and numeracy. Neuroimage 118:79-89.

Carreiras M, Quiñones I, Hernández-Cabrera JA, Duñabeitia JA (2015b) Orthographic coding: brain activation for letters, symbols, and digits. Cereb Cortex 25:4748-4760.

Cavanna AE, Trimble MR (2006) The precuneus: a review of its functional anatomy and behavioural correlates. Brain 129:564-583.

Chen Y, Davis MH, Pulvermüller F, Hauk O (2013) Task modulation of brain responses in visual word recognition as studied using EEG/MEG and fMRI. Front Hum Neurosci 7:376.

Chen Y, Huang L, Chen K, Ding J, Zhang Y, Yang Q, Lv Y, Han Z, Guo Q (2020) White matter basis for the hub-and-spoke semantic representation: evidence from semantic dementia. Brain 143:1206-1219.

Cibelli ES, Leonard MK, Johnson K, Chang EF (2015) The influence of lexical statistics on temporal lobe cortical dynamics during spoken word listening. Brain Lang 147:66-75.

Cohen L, Dehaene S, Vinckier F, Jobert A, Montavont A (2008) Reading normal and degraded words: contribution of the dorsal and ventral visual pathways. Neuroimage 40:353-366.

Coltheart M, Saunders SJ, Tree JJ (2010) Computational modelling of the effects of semantic dementia on visual word recognition. Cogn Neuropsychol 27:101-114.

Dehaene S, Cohen L (2011) The unique role of the visual word form area in reading. Trends Cogn Sci 15:254-262.

Delorme A, Makeig S (2004) EEGLAB: an open source toolbox for analysis of single-trial EEG dynamics including independent component analysis. J Neurosci Methods 134:9-21.

De Putte EV, De Baene W, Price CJ, Duyck W (2018) Neural overlap of L1 and $\mathrm{L} 2$ semantic representations across visual and auditory modalities: a decoding approach. Neuropsychologia 113:68-77.

Dickens JV, Fama ME, DeMarco AT, Lacey EH, Friedman RB, Turkeltaub PE (2019) Localization of Phonological and Semantic Contributions to Reading. J Neurosci 39:5361-5368.

Ding J, Chen K, Liu H, Huang L, Chen Y, Lv Y, Yang Q, Guo Q, Han Z, Lambon Ralph MA (2020) A unified neurocognitive model of semantics language social behaviour and face recognition in semantic dementia. Nat Commun 11:2595.

Edwards JD, Pexman PM, Goodyear BG, Chambers CG (2005) An fMRI investigation of strategies for word recognition. Cognitive Brain Res 24:648-662. 
Faul F, Erdfelder E, Lang AG, Buchner A (2007) G*Power 3: a flexible statistical power analysis program for the social, behavioral, and biomedical sciences. Behav Res Methods 39:175-191.

Ferstl EC, Neumann J, Bogler C, von Cramon DY (2008) The extended language network: a meta-analysis of neuroimaging studies on text comprehension. Hum Brain Mapp 29:581-593.

Finn ES, Shen X, Holahan JM, Scheinost D, Lacadie C, Papademetris X, Shaywitz SE, Shaywitz BA, Constable RT (2014) Disruption of functional networks in dyslexia: a whole-brain, data-driven analysis of connectivity. Biol Psychiatry 76:397-404.

Fischl B (2012) FreeSurfer. Neuroimage 62:774-781.

Gow DW Jr, Segawa JA (2009) Articulatory mediation of speech perception: a causal analysis of multi-modal imaging data. Cognition 110:222-236.

Gow DW Jr, Segawa JA, Ahlfors SP, Lin F-H (2008) Lexical influences on speech perception: a Granger causality analysis of MEG and EEG source estimates. Neuroimage 43:614-623.

Gitelman DR, Nobre AC, Sonty S, Parrish TB, Mesulam MM (2005) Language network specializations: an analysis with parallel task designs and functional magnetic resonance imaging. Neuroimage 26:975-985.

Hauk O, Patterson K, Woollams A, Watling L, Pulvermüller F, Rogers TT (2006) [Q:] When would you prefer a SOSSAGE to a SAUSAGE? [A:] At about $100 \mathrm{msec}$. ERP correlates of orthographic typicality and lexicality in written word recognition J of Cogn Neurosci 18:818-832.

Hauk O, Coutout C, Holden A, Chen Y (2012) The time-course of singleword reading: evidence from fast behavioral and brain responses. Neuroimage 60:1462-1477.

Hirshorn EA, Li Y, Ward MJ, Richardson RM, Fiez JA, Ghuman AS (2016) Decoding and disrupting left mid-fusiform gyrus activity during word reading. Proc Natl Acad Sci U S A 113:8162-8167.

Jobard G, Crivello F, Tzourio-Mazoyer N (2003) Evaluation of the dual route theory of reading: a meta analysis of 35 neuroimaging studies. Neuroimage 20:693-712.

Kadipasaoglu CM, Baboyan VG, Conner CR, Chen G, Saad ZS, Tandon N (2014) Surface-based mixed effects multilevel analysis of grouped human electrocorticography. Neuroimage 101:215-224.

Klein M, Grainger J, Wheat KL, Millman RE, Simpson MI, Hansen PC, Cornelissen PL (2015) Early activity in Broca's area during reading reflects fast access to articulatory codes from print. Cereb Cortex 25:1715-1723.

Lachaux JP, Axmacher N, Mormann F, Halgren E, Crone NE (2012) Highfrequency neural activity and human cognition: past, present and possible future of intracranial EEG research. Prog Neurobiol 98:279-301.

Lau EF, Phillips C, Poeppel D (2008) A cortical network for semantics: (De) constructing the N400. Nat Rev Neurosci 9:920-933.

Levy J, Pernet C, Treserras S, Boulanouar K, Aubry F, Démonet JF, Celsis P (2009) Testing for the dual-route cascade reading model in the brain: an fMRI effective connectivity account of an efficient reading style. PLoS One 4:e6675.

Li M, Xu Y, Luo X, Zeng J, Han Z (2020) Linguistic experience acquisition for novel stimuli selectively activates the neural network of the visual word form area. Neuroimage 215:116838.

Li Y, Kang JS (1993) Analysis of phonetics of the ideophonetic characters in modern Chinese. In: Information analysis of usage of characters in modern Chinese. (Chen Y, ed), pp 84-98. Shanghai: Shanghai Education.

Lin SE, Chen HC, Zhao J, Li S, He S, Weng XC (2011) Left-lateralized N170 response to unpronounceable pseudo but not false Chinese charactersthe key role of orthography. Neuroscience 190:200-206.

Lochy A, Jacques C, Maillard L, Colnat-Coulbois S, Rossion B, Jonas J (2018) Selective visual representation of letters and words in the left ventral occipito-temporal cortex with intracerebral recordings. Proc Natl Acad Sci U S A 115:E7595- 7604

Makeig S, Debener S, Onton J, Delorme A (2004) Mining event-related brain dynamics. Trends in Cognitive Sciences 8:204-210.

Mano QR, Humphries C, Desai RH, Seidenberg MS, Osmon DC, Stengel BC, Binder JR (2013) The role of left occipitotemporal cortex in reading: reconciling stimulus, task, and lexicality effects. Cereb Cortex 23:988-1001.

Matsuo T, Kawasaki K, Kawai K, Majima K, Masuda H, Murakami H, Kunii N, Kamitani Y, Kameyama S, Saito N, Hasegawa I (2015) Alternating zones selective to faces and written words in the human ventral occipitotemporal cortex. Cereb Cortex 25:1265-1277.

Nobre AC, Allison T, McCarthy G (1994) Word recognition in the human inferior temporal lobe. Nature 372:260-263.
Oldfield RC (1971) The assessment and analysis of handedness: the Edinburgh inventory. Neuropsychologia 9:97-113.

Omigie D, Dellacherie D, Hasboun D, George N, Clement S, Baulac M, Adam C, Samson S (2015) An intracranial EEG study of the neural dynamics of musical valence processing. Cereb Cortex 25:4038-4047.

Oostenveld R, Fries P, Maris E, Schoffelen J (2011) FieldTrip: open source software for advanced analysis of MEG, EEG, and invasive electrophysiological data. Comput Intell Neurosci 2011:1-9.

Osman A, Bashore TR, Coles MGH, Donchin E, Meyer DE (1992) On the transmission of partial information: inferences from movement-related brain potentials. J Exp Psychol Hum Percep Perform 18:217-232.

Ossmy O, Ben-Shachar M, Mukamel R (2014) Decoding letter position in word reading. Cortex 59:74-83.

Ozker M, Schepers IM, Magnotti JF, Yoshor D, Beauchamp MS (2017) A double dissociation between anterior and posterior superior temporal gyrus for processing audiovisual speech demonstrated by electrocorticography. J Cogn Neurosci 29:1044-1060.

Pammer K, Hansen P, Holliday I, Cornelissen P (2006) Attentional shifting and the role of the dorsal pathway in visual word recognition. Neuropsychologia 44:2926-2936.

Perrone-Bertolotti M, Kujala J, Vidal JR, Hamame CM, Ossandon T, Bertrand OF, Minotti L, Kahane P, Jerbi K, Lachaux J (2012) How silent is silent reading? Intracerebral evidence for top-down activation of temporal voice areas during reading. J Neurosci 32:1755417562.

Price CJ (2012) A review and synthesis of the first 20 years of PET and fMRI studies of heard speech, spoken language and reading. Neuroimage 62:816-847.

Price CJ, Devlin JT (2011) The interactive account of ventral occipitotemporal contributions to reading. Trends Cogn Sci 15:246-253.

Price CJ, Mechelli A (2005) Reading and reading disturbance. Curr Opin Neurobiol 15:231-238.

Purcell JJ, Shea J, Rapp B (2014) Beyond the visual word form area: the orthography-semantics interface in spelling and reading. Cogn Neuropsychol 31:482-510.

Rämä P, Sala JB, Gillen JS, Pekar JJ, Courtney SM (2001) Dissociation of the neural systems for working memory maintenance of verbal and nonspatial visual information. Cogn Affect Behav Neurosci 1:161-171.

Rypma B, Prabhakaran V, Desmond JE, Glover GH, Gabrieli J (1999) Loaddependent roles of frontal brain regions in the maintenance of working memory. NeuroImage 9:216-226.

Seth AK (2005) Causal connectivity of evolved neural networks during behavior. Network 16:35-54.

Shu H, Chen X, Anderson RC, Wu N, Xuan Y (2003) Properties of school Chinese: implications for learning to read. Child Dev 74:27-47.

Si X, Zhou W, Hong B (2017) Cooperative cortical network for categorical processing of Chinese lexical tone. Proc Natl Acad Sci U S A 114:1230312308 .

Solomyak O, Marantz A (2009) Lexical access in early stages of visual word processing: a single-trial correlational MEG study of heteronym recognition. Brain Lang 108:191-196.

Taft M, Zhu X, Peng D (1999) Positional specificity of radicals in Chinese character recognition. J Mem Lang 40:498-519.

Tan LH, Feng CM, Fox PT, Gao JH (2001) An fMRI study with written Chinese. NeuroReport 12:83-88.

Tan LH, Laird AR, Li K, Fox PT (2005) Neuroanatomical correlates of phonological processing of Chinese characters and alphabetic words: a metaanalysis. Hum Brain Mapp 25:83-91.

Taylor JSH, Rastle K, Davis MH (2013) Can cognitive models explain brain activation during word and pseudoword reading? A meta-analysis of 36 neuroimaging studies. Psychological Bulletin 139:766-791.

Thesen T, Mcdonald CR, Carlson C, Doyle W, Cash S, Sherfey JS, Felsovalyi O, Girard HM, Barr WB, Devinsky O, Kuzniecky R, Halgren E (2012) Sequential then interactive processing of letters and words in the left fusiform gyrus. Nat Commun 3:1284.

Townsend JT, Ashby FG (1983) Stochastic modeling of elementary psychological processes. New York: Cambridge UP.

Tzourio-Mazoyer N, Landeau B, Papathanassiou D, Crivello F, Etard O, Delcroix N, Mazoyer B, Joliot M (2002) Automated anatomical labeling of activations in SPM using a macroscopic anatomical parcellation of the MNI MRI single-subject brain. Neuroimage 15:273-289. 
Vinckier F, Dehaene S, Jobert A, Dubus JP, Sigman M, Cohen L (2007) Hierarchical coding of letter strings in the ventral stream: dissecting the inner organization of the visual word-form system. Neuron 55:143-156.

Wagenmakers E-J, Marsman M, Jamil T, Ly A, Verhagen J, Love J, Selker R, Gronau QF, Šmíra M, Epskamp S, Matzke D, Rouder JN, Morey RD (2018) Bayesian inference for psychology. Part I: theoretical advantages and practical ramifications. Psychon Bull Rev 25:35-57.

Wang X, Zhao R, Zevin JD, Yang J (2016) The neural correlates of the interaction between semantic and phonological processing for Chinese character reading. Front Psychol 7:518.

Weekes BS, Yin W, Su I, Chen M (2006) The cognitive neuropsychology of reading and writing in Chinese. Lang Linguis 7:595-617.

Wei T, Liang X, He Y, Zang Y, Han Z, Caramazza A, Bi Y (2012) Predicting conceptual processing capacity from spontaneous neuronal activity of the left middle temporal gyrus. J Neurosci 32:481-489.

Whaley ML, Kadipasaoglu CM, Cox SJ, Tandon N (2016) Modulation of orthographic decoding by frontal cortex. J Neurosci 36:11731184.

Wheat KL, Cornelissen PL, Frost SJ, Hansen PC (2010) During visual word recognition, phonology is accessed within $100 \mathrm{~ms}$ and may be mediated by a speech production code: evidence from Magnetoencephalography. J Neurosci 30:5229-5233.
Woodhead ZVJ, Barnes GR, Penny W, Moran R, Teki S, Price CJ, Leff AP (2014) Reading front to back: MEG evidence for early feedback effects during word recognition. Cereb Cortex 24:817-825.

Woollams AM, Silani G, Okada K, Patterson K, Price CJ (2011) Word or word-like? Dissociating orthographic typicality from lexicality in the left occipito-temporal cortex. J Cogn Neurosci 23:992-1002.

Woolnough O, Donos C, Rollo PS, Forseth KJ, Lakretz Y, Crone NE, Fischer-Baum S, Dehaene S, Tandon N (2021) Spatiotemporal dynamics of orthographic and lexical processing in the ventral visual pathway. Nat Hum Behav 5:389-398.

Wu C, Ho MR, Chen SA (2012) A meta-analysis of fMRI studies on Chinese orthographic, phonological, and semantic processing. Neuroimage 63:381-391.

Xia M, Wang J, He Y (2013) BrainNet Viewer: a network visualization tool for human brain connectomics. PLoS One 8:e68910.

Yang J, Wang X, Shu H, Zevin JD (2011) Brain networks associated with sublexical properties of Chinese characters. Brain Lang 119:68-79.

Yum YN, Su IF, Law SP (2015) Early effects of radical position legality in Chinese: An ERP study. Sci Stud Read 19:456-467.

Yum YN, Law S-P, Lee CF, Shum MSK (2017) Early event-related potentials differences in orthographic processing of native and non-native Chinese readers. J Res Read 41:403-422.

Zhang B, He S, Weng X (2018) Localization and functional characterization of an occipital visual word form sensitive area. Sci Rep 8:6723. 